\title{
As estratégias de coping do trabalhador da enfermagem hospitalar
}

\author{
Coping strategies of hospital nursing workers \\ Estrategias de afrontamiento de los trabajadores de enfermería hospitalaria
}

\section{Resumo}

Objetivo: avaliar as estratégias de enfrentamento de coping de trabalhadores de enfermagem de um hospital universitário. Método: estudo transversal, realizado em 2018 com 67 trabalhadores de enfermagem. Para a avaliação das estratégias de enfrentamento foi utilizado a Escala de Coping Ocupacional. Resultados: a maioria eram mulher cisgênero, solteiras, com média de idade de 39,1 anos, técnicos de enfermagem e atuavam no turno diurno, sendo que a população apresentou o processo de coping por meio de estratégia de enfrentamento controle (média de 3,58). Conclusão: que o conhecimento sobre as estratégias de coping favorece o gerenciamento de recursos humanos, detecção precoce de problemas relacionados ao ambiente laboral, além da prevenção de riscos ao trabalhador e promoção da saúde laboral.

Palavras-chave: Saúde do trabalhador; Condições de trabalho; Estresse ocupacional; Saúde pública.

\begin{abstract}
Objective: to evaluate coping coping strategies of nursing workers at a university hospital. Method: cross-sectional study carried out in 2018 with 67 nursing workers. To assess coping strategies, the Occupational Coping Scale was used. Results: most were cis-gender women, single, with a mean age of 39.1 years, nursing technicians and working the day shift, and the population presented the coping process through a control coping strategy (average of 3,58). Conclusion: that knowledge about coping strategies favors the management of human resources, early detection of problems related to the work environment, in addition to the prevention of risks to workers and promotion of occupational health.
\end{abstract}

Keywords: Occupational health; Work conditions; Occupational stress; Public health.

\section{Resumen}

Objetivo: evaluar las estrategias de afrontamiento de los trabajadores de enfermería de un hospital universitario. Método: estudio transversal realizado en 2018 con 67 trabajadores de enfermería. Para evaluar las estrategias de afrontamiento se utilizó la Escala de afrontamiento ocupacional. Resultados: la mayoría fueron mujeres cisgénero, solteras, con una edad media de 39,1 años, técnicas de enfermería y en jornada diurna, y la población presentó el proceso de afrontamiento a través de una estrategia de afrontamiento de control (promedio de 3, 58). Conclusión: que el conocimiento sobre estrategias de afrontamiento favorece la gestión de los recursos humanos, la detección precoz de problemas relacionados con el entorno laboral, además de la prevención de riesgos para los trabajadores y la promoción de la salud ocupacional.

Palabras clave: Salud laboral; Condiciones de trabajo; Estrés laboral; Salud pública.

\section{Introdução}

As estratégias de coping são recursos cognitivos, emocionais e comportamentais que o indivíduo emprega na tentativa de lidar com situações estressoras (Antonioli et al., 2018).

O processo de coping envolve quatro características principais: interação do indivíduo com o ambiente; administração 
da situação estressora, em vez de controle ou domínio da mesma; avaliação da situação, ou seja, como o fenômeno é percebido, interpretado e cognitivamente representado na vida do indivíduo e mobilização de esforços, através dos quais os indivíduos irão empreender ações cognitivas e comportamentais para administrar as demandas internas ou externas que surgem da sua interação com o ambiente (Dias et al., 2020).

Todo profissional em contato direto com a clientela fica suscetível ao estresse no trabalho. Há, portanto, imperiosa necessidade de que os profissionais de saúde tenham o conhecimento suficiente para poderem gerenciar as situações estressoras a partir das estratégias de coping (Luz et al., 2017).

Desse modo, o presente estudo foi realizado com o objetivo de avaliar as estratégias de coping de trabalhadores de enfermagem de um hospital universitário brasileiro.

\section{Metodologia}

O projeto de pesquisa foi submetido à análise pelo Comitê de Ética em Pesquisa com Seres Humanos da Universidade Federal de São Carlos, com parecer de aprovação nº 3.043.333 e CAAE: 88169218.8.0000.5504.

Trata-se de um estudo quantitativo, analítico, transversal, (Gil, 2010) realizado em um Hospital Universitário vinculado a uma instituição pública de ensino superior, que fica localizado no interior de São Paulo, Brasil.

A amostra não probabilística intencional deste estudo foi composta por 35 técnicos de enfermagem e 32 enfermeiros (67 trabalhadores) que concordaram em participar do estudo mediante convite realizado no local de trabalho. Foram excluídos do estudo enfermeiros e técnicos de enfermagem que estavam de licença saúde, licença maternidade ou de férias no momento da coleta de dados.

Para avaliação das estratégias de enfrentamento coping, foi utilizado a Escala de Coping Ocupacional, que consiste na versão brasileira que foi traduzida e adaptada por Pinheiro et al., (2000) e proposta por Latack (1986). A escala apresenta os fatores controle, esquiva e manejo de sintomas; é composta de 29 itens, sendo que 11 estão relacionados ao controle, 9 a esquiva e 9 itens ao manejo do estresse com opções de intensidade que variam "nunca faço isso" (1), "raramente faço isso" (2), "às vezes faço isso" (3), "frequentemente faço isso" (4) a "sempre faço isso" (5). Os escores de cada fator classificatório da escala são realizados pela média dos itens que os compõe. Assim, o fator que apresentar maior média será considerado o prevalente para cada trabalhador (Pinheiro et al., 2003).

Para a análise dos dados utilizou-se o Programa Statistical Package of Social Sciences (SPSS) versão 21.0. Foi realizada dupla digitação dos dados em planilha de Excell for Windows 8. A consistência interna da escala da Escala de Coping Ocupacional foi avaliada por meio do Alfa de Cronbach. Para a construção das tabelas optou-se pela estatística descritiva, com apresentação das variáveis categóricas em frequência absoluta e relativa. Foi realizado o Teste $\mathrm{T}$ de comparação de grupos, fixando-se um nível de significância de 5\%.

\section{Resultados e Discussão}

O alfa de Cronbach para Escala de Coping Ocupacional foi superior a 0,7 o que sugere a confiabilidade interna do instrumento.

Em relação a caracterização da amostra, $80,6 \%$ se declararam como mulher cisgênero, 54,4\% solteiros, a média de idade foi de 39,1 7,09 anos, 52,9\% eram técnicos de enfermagem, 89,7\% atuavam em uma carga horária de 36 horas e 60,3\% pertenciam ao turno diurno.

Na Tabela 1, apresentamos os resultados das dimensões do coping por categoria profissional e suas respectivas comparações. 
Tabela 1 - Descritiva das dimensões do coping por categoria profissional e teste de comparação entre as categorias. São Paulo, Brasil, 2018. ( $\mathrm{N}=67)$.

\begin{tabular}{cccccc}
\hline $\begin{array}{c}\text { Categoria } \\
\text { Profissional }\end{array}$ & Controle & Esquiva & $\begin{array}{c}\text { Manejo de } \\
\text { Sintomas }\end{array}$ & Valor p \\
\hline Técnico de & $\mathrm{n}$ & 35 & 35 & 35 & $0,000^{*}$ \\
Enfermagem & Média & $3,58^{\mathrm{a}}$ & 2,65 & 2,51 & \\
& Mediana & 3,64 & 2,67 & 2,56 & $0,000^{*}$ \\
Enfermeiro & Desvio padrão & 0,68 & 0,61 & 0,72 & 32 \\
& Média & 32 & 32 & 2,73 & 2,67 \\
& Mediana & $3,58^{\mathrm{a}}$ & 2,67 & 0,79 & \\
& Desvio padrão & 0,57 & 2,61 & 0,51 & $0,236^{* *}$ \\
\hline
\end{tabular}

* Teste ANOVA / **Teste t. Fonte: Elaborado pelos autores.

Ao realizar a comparação, foi verificado que não existe diferença entre os grupos dentro de cada dimensão (todos os valores de p são superiores a 0,05). Porém, quando se comparam as dimensões dentro de cada grupo observa-se que a dimensão controle tem valores mais altos estatisticamente $(p=0,000)$ tanto entre os técnicos de enfermagem quanto entre os enfermeiros.

Na Tabela 2, apresentamos as dimensões do copign e sua comparação entre gêneros.

Tabela 2 - Descritiva das dimensões do coping por gênero e teste de comparação entre os gêneros. São Paulo, Brasil, 2018. $(\mathrm{N}=67)$.

\begin{tabular}{ccccc}
\hline Gênero & & Controle & Esquiva & Manejo de Sintomas \\
\hline Mulher & $\mathrm{n}$ & 53 & 53 & 53 \\
Cisgênero & Média & 3,73 & 2,67 & 2,56 \\
& Mediana & 0,63 & 0,55 & 0,78 \\
& Desvio padrão & 2,00 & 1,00 & 1,00 \\
Homem & $\mathrm{n}$ & 13 & 13 & 13 \\
Cisgênero & Média & 3,66 & 2,66 & 2,81 \\
& Mediana & 3,82 & 2,67 & 2,89 \\
& Desvio padrão & 0,54 & 0,64 & 0,66 \\
& Valor p* & $0,663 *$ & $0,953^{*}$ & $0,324 *$ \\
\hline
\end{tabular}

*Teste t. Fonte: Elaborado pelos autores.

Ao comparar as dimensões por gênero observa-se que não existe diferença entre mulher cisgênero e homem cisgênero em nenhuma das dimensões (valores de p são superiores a 0,05).

Ficou evidenciado nesta pesquisa que a equipe de enfermagem realiza ações e reavaliações cognitivas proativas como estratégias de enfrentamento para administrar situações estressoras (controle) como a resolução de problemas. Nesse tipo de enfrentamento das situações estressoras do processo de trabalho, o profissional opta por se mobilizar para enfrentar a situação estressora e gerencia-la.

As estratégias de enfrentamento são descritas na literatura como "um conjunto de ações de ordem cognitiva, comportamental ou emocional, para controlar situações de estresse e manter a integridade mental e física” (Dalbosco, 2020, p. $6)$. 
Existem duas categorias de estratégias de enfrentamento, sendo elas, o enfrentamento focado na emoção e o enfrentamento focado no problema. No enfrentamento focado na emoção, o trabalhador busca controlar as emoções desencadeadas nos eventos estressantes do ambiente laboral, como por exemplo, buscando verbalizar suas emoções com um colega de trabalho. Já no enfrentamento focado nos problemas, o trabalhador busca de forma imediata solucionar a situações estressora por meio da fonte, como por exemplo, buscando orientação ou um responsável direto que pode dar resolutividade para a situação. (Santos et al., 2016).

A estratégia reavaliação positiva refere-se às estratégias cognitivas para aceitação da realidade, em que o indivíduo procura aspectos que aliviem a situação estressante ou se foca nos aspectos positivos para reduzir a carga emotiva da situação, redirecionando o estressor (Souza et al., 2018).

Observou-se que a dimensão do coping controle foi a prevalente entre a equipe de enfermagem de modo semelhante a outro estudo brasileiro. Esse resultado reforça os resultados encontrados de que esses sujeitos avaliam os estressores no trabalho como eventos positivos e usam estratégias de enfrentamento adequadas a essas situações (Andolhe et al., 2015).

Em um estudo desenvolvido com técnicos de enfermagem de um Pronto Socorro foi identificado que a ajuda mútua e o trabalho em equipe configuraram-se como estratégias de enfrentamento que visaram diminuir a carga de trabalho deste setor. Ainda, pontuaram que o não envolvimento com os pacientes, separar a vida profissional do pessoal, a espiritualidade/religiosidade, o planejamento de ações no setor e o reconhecimento foram fatores que colaboraram no enfrentamento das adversidades e do sofrimento (Monteiro et al., 2013).

Em estudo realizado com enfermeiros de terapia intensiva em um hospital público do Rio Grande do Sul, foi observado que os enfermeiros enfrentam as situações de estresse de uma maneira proativa no ambiente de trabalho, ou seja, promovem reavaliações cognitivas a respeito dos estressores e da forma de reagir diante deles. Ainda, destaca-se que o enfrentamento consiste em uma ação intencional, de ordem física e psíquica, direcionado a circunstâncias extrínsecas ou intrínsecas em resposta a um agente estressor verificado (Kleinubing et al., 2013).

Portanto, a equipe de enfermagem identifica as situações problemáticas no ambiente de trabalho e tende a agir, utilizando esforços cognitivos e comportamentais, no intuito de compreender e administrar a origem do problema. Neste sentido, a maior utilização de respostas de enfrentamento corresponderia a atitudes positivas e ativas frente as dificuldades no trabalho, o que contribuiria no gerenciamento do estresse ocupacional, com repercussões benéficas para o desempenho profissional, preservação da saúde e qualidade da assistência (Jacques et al., 2017).

Em contrapartida, estudo realizado em unidade de hemodiálise na Austrália, com o objetivo de verificar as estratégias de coping utilizadas pelos enfermeiros, identificou maior utilização do coping centrado na emoção. Isto é, utilização de respostas de evitação, em que o indivíduo tenta reestruturar o acontecimento com o intuito de encontrar aspectos favoráveis para amenizar a gravidade do estressor, concentrando-se nos aspectos positivos como forma de amenizar a carga emotiva negativa da situação, como sentimento de dever cumprido após a realização das rotinas de cuidado, busca de apoio espiritual, e conversar sobre assuntos não referentes ao trabalho (Dolan et al., 2012).

Ao se analisar as estratégias de enfrentamento, é importante pontuar que o processo pode incluir tanto respostas positivas sobre o agente estressor, como respostas negativas. Deve-se também destacar que uma estratégia de coping não pode ser considerada como intrinsecamente adaptativa, tornando-se necessário considerar a natureza desse agente, a disponibilidade de recursos e o resultado do esforço individual (Bublitz et al., 2015).

Ao tratar sobre a correlação entre a utilização das estratégias de enfrentamento e o estresse, observa-se que o uso do coping aumenta conforme o nível total de estresse aumenta no ambiente laboral. Como consequência, ele pode desenvolver no trabalhador um desgaste físico e psíquico (Bezerra et al., 2016).

Cabe ressaltar que as diferentes situações de estresse podem influenciar na escolha das estratégias utilizadas, ou seja, 
dependendo do agente e da sua avaliação, será determinada pelo indivíduo uma resposta ou a união de duas ou mais estratégias que podem ser utilizadas em conjunto (Kleinubing et al., 2013).

Os achados sugerem que a equipe de enfermagem pesquisada no estudo optou por utilizar as estratégias de coping focadas na resolução dos problemas no ambiente de trabalho, sendo essas respostas positivas para o crescimento da equipe enquanto profissional e pessoal.

Logo, cabe citar que, quando essas estratégias são utilizadas de forma efetiva, os profissionais de enfermagem tendem a diminuir ou solucionar os problemas provocados pelos agentes estressores (Bublitz et al., 2015).

\section{Conclusão}

A preocupação com o estresse e o seu gerenciamento vem ganhando força em virtude das profundas transformações sociais que atingem o ambiente de trabalho e os profissionais que ali estão, podendo gerar tensões, afastamentos, sobrecargas físicas, psíquicas e adoecimentos.

Em relação às estratégias de enfrentamento, sobressaiu o controle, ou seja, o trabalhador percebe as demandas do ambiente e se mobiliza para tentar modificar a situação estressante como forma de enfrentamento sendo ações e reavaliações cognitivas proativas. As estratégias de controle foram avaliadas como eficazes para o enfrentamento do estresse.

As estratégias de enfrentamento dependem das características individuais do profissional e das situações vivenciadas no ambiente ocupacional. Por conseguinte, a adoção de diversas estratégias é mais eficaz do que o uso de apenas uma, visto que o indivíduo tem mais recursos para enfrentar a situação estressante ao aderir a várias delas.

Destarte, salienta-se que o conhecimento sobre as estratégias de coping favorece o gerenciamento de recursos humanos, detecção precoce de problemas relacionados ao ambiente laboral, além da prevenção de riscos ao trabalhador e promoção da saúde laboral.

\section{Referências}

Andolhe, R., Barbosa, R. L., Oliveira, E. M., Costa, A. L. S. \& Padilha, K. G. (2015). Estresse, coping, e burnout da equipe de enfermagem de Unidade de Terapia Intensiva: fatores associados. Rev Esc Enferm USP. 49(Esp.):58-64.

Antoniolli, L., Echevarría-Guanilo, M. E., Rosso, L. H., Fuculo Junior, P. R. B., Dal Pai, D. \& Scapin, S. (2018). Estratégias de coping da equipe de enfermagem atuante em centro de tratamento ao queimado. Rev. Gaúcha Enferm. 39: e2016-0073.

Benetti, E. R. R., Stumm, E.M.F., Weiller, T. H., Batista, K. M., Lopes, L. F. D. \& Guido, L. A. (2015). Estratégias de coping e características de trabalhadores de enfermagem de hospital privado. Rev Rene 16(1):3-10.

Bezerra, C. M., Assis, S. G. \& Constantino, P. (2016). Psychological distress and work stress in correctional officers: a literature review. Ciênc Saúde Coletiva 21(7):2135-46.

Bublitz, S., Guido, L. A., Lopes, L. F. D. \& Freitas, E. O. (2015) Association between nursing students academic and sociodemographic characteristics and stress. Texto Contexto Enferm 25(4):e2440015.

Dal'Bosco, E. B., Floriano, L. S. M., Skupien, S. V., Arcaro, G., Martins, A. R. \& Anselmo, A. C. C. (2020). A saúde mental da enfermagem no enfrentamento da COVID-19 em um hospital universitário regional. Rev. Bras. Enferm. 73(2): e20200434.

Dias, E. N. \& Pais-Ribeiro, J. L. (2019). O modelo de coping de Folkman e Lazarus: aspectos históricos e conceituais. Rev. Psicol. Saúde 11(2): 55-66.

Dolan, G., Strodl, E. \& Hamernik, E. (2012). Why renal nurses cope so well with their workplace stressors. J Ren Care 38(4):222-32.

Gil, A. C. (2010). Como elaborar projetos de pesquisa. (5a ed.) Atlas.

Jacques, J. P. B., Oussak, L. C. S., Scholze, A. R., Ribeiro, B. G. A., Martins, J. T., \& Perfeito, R. R. (2017). Personalidade hardiness ecoping entre profissionais de enfermagem do centro cirúrgico. Rev enferm UFPE online 11(11).

Kleinubing, R. E., Goulart, C. T., Silva, R. M., Umann, J., \& Guido, L. A. (2013). Estresse e coping em enfermeiros de terapia intensiva adulto e cardiológica. Rev Enferm UFSM 3(2):335-44.

Luz, L. M., Torres, R. R. B., Sarmento, \& K. M. V. Q. et al. (2017). Síndrome de Burnout em profissionais do serviço de atendimento móvel de urgência. Rev Fund Care Online.; 9(1):238-246. 
Research, Society and Development, v. 11, n. 2, e47811225282, 2022

(CC BY 4.0) | ISSN 2525-3409 | DOI: http://dx.doi.org/10.33448/rsd-v11i2.25282

Monteiro, J. K., Oliveira, A. L. L., Ribeiro, C. S., Grisa, G. H., Agostini, N. (2013). Adoecimento Psíquico de Trabalhadores de Unidades de Terapia Intensiva. Psicol Ciênc Prof 33(2):366-79.

Ribeiro, R. M., Pompeo, D. A., Pinto, M. H., Ribeiro, \& R. C. H. M. (2015). Estratégias de enfrentamento dos enfermeiros em serviço hospitalar de emergência. Acta Paul Enferm 28(3):216- 23.

Pinheiro, F. A., Tróccoli, B. T, \& Tamayo, M. R. (2003). Mensuração de coping no ambiente ocupacional. Psicologia: Teoria e Pesquisa, 19(2), 153-158.

Santos, N. A. R., Santos, A. T C., \& Silva, R. P. (2016). Coping strategies of nurses in the care of patients with head and neck neoplasms. Rev. esc. enferm. USP 50(4): 569-578.

Souza, S. B. C., Milioni, K. C., \& Dornelles, T. M. (2018). Análise do grau de complexidade do cuidado, estresse e coping da enfermagem num hospital sulriograndense. Texto contexto - enferm. 27(4): e4150017.

Souza, R. C., Silva, S. M., \& Costa, M. L. A. S. (2018). Estresse ocupacional no ambiente hospitalar: revisão das estratégias de enfrentamento dos trabalhadores de enfermagem. Rev. bras. med. trab; 16(4): 493-502.

Souza, S. S., Borenstein, M. S., Silva, D. M. G. V., Souza, S. S., \& Carvalho, J. B. (2013). Estratégias de enfrentamento da enfermagem no cuidado ao potencial doador de órgãos. Rev Rene 14(1):92- 100.

Tamayo, M. R., Pinheiro, F. A., Tróccoli, B. T., \& Paz, M. G. T. (2000). Construção e validação da escala de suporte organizacional percebido (ESOP) [Resumo]. Anais da Reunião Anual da Sociedade Brasileira de Psicologia (p. 52). Brasília: Sociedade Brasileira de Psicologia.

Umann, J., Silva, R. M., Benavente, S. B. T., \& Guido, L. A. (2014). O impacto das estratégias de enfrentamento na intensidade de estresse de enfermeiras de hemato-oncologia. Rev Gaúcha Enferm 35(3):103-10. 